\title{
Toluidine Blue 0-Gelatin Gel Dosimeter for Radiation Processing
}

\author{
S. M. Gafar, M. A. El-Kelany, M. A. El-Ahdal, S. R. El-Shawadfy \\ National Center for Radiation Research and Technology, AEA, Cairo, Egypt \\ Email: dr.moshy@gmail.com
}

Received 1 June 2014; revised 3 July 2014; accepted 15 July 2014

Copyright (C) 2014 by authors and Scientific Research Publishing Inc.

This work is licensed under the Creative Commons Attribution International License (CC BY).

http://creativecommons.org/licenses/by/4.0/

c. (i) Open Access

\begin{abstract}
In this study the spectrophotometric response of the Toluidine Blue 0 (TBO)-gelatin gel dosimeter irradiated with gamma rays was characterized. Preparation of dyed-gelatin gel takes place in neutral medium; to give eventually gel dosimeter has a sharp absorbance peak at $635 \mathrm{~nm}$ that bleaches quantitatively upon irradiation and the colour change can be measured with UV-VIS spectrophotometer. The useful dose range was 1 - $150 \mathrm{~Gy}$. The radiation chemical yield (G-value) of gel dosimeter was calculated and found to increase by increasing concentration of dye. Post-irradiation storage on the response of gel is discussed. The dose response function, radiation sensitivity, and dependences of the response on environmental factors were studied.
\end{abstract}

\section{Keywords}

\section{Gel, Toluidine Blue 0, Dosimeter, $\gamma$ Rays and Low Dose}

\section{Introduction}

Ionizing radiation energy absorbed in the conventional Fricke solution [1] [2] causes oxidation of ferrous ions $\left(\mathrm{Fe}^{2+}\right)$ into ferric ions $\left(\mathrm{Fe}^{3+}\right)$ [3] proposed incorporating the Fricke solution into a gel matrix to spatially stabilize the dosimetric information so that the dose distribution could potentially be measured in three dimensions. With the addition of the metal ion indicator xylenol orange (XO), $\mathrm{Fe}^{3+}$ binds to $\mathrm{XO}$ forming a 1:1 colored complex $\left(\mathrm{XO}-\mathrm{Fe}^{3+}\right)$ in the visible range that can be measured spectrophotometrically [4]. The irradiated-dose distribution in Fricke-type gel dosimeters has traditionally been evaluated with magnetic resonance imaging (MRI) [5] for the purpose of 3D radiotherapy dosimetry. However, as the resulting irradiated-dose distribution diffuses over time, the evaluation technique is time-limited due to the resulting blurred dose distribution. Alternative optical evaluation techniques (Laser-scanned agarose gel sections for radiation field mapping) [6]. Hydrogels can be obtained in various ways. The following methods are most widely employed: chemical cross-linking [7] mostly 
using glutaraldehyde as the cross-linking agent [8]; cross-linking by $\gamma$-radiation [9] [10], by UV radiation [11], and by use of successive freezing/thawing cycles [12] [13]. Intermolecular bonds (mostly hydrogen bonds), which are formed during the freezing/thawing process of PVA water solutions, act as efficient cross-links [14]. Gelatin has the advantage of dissolving at a lower temperature (about $41^{\circ} \mathrm{C}$ ) than agarose (about $90^{\circ} \mathrm{C}$ ), meaning less oxygen is lost during heating that agarose is translucent, whereas gelatin is transparent, which is very important for optical absorbance measurements [15]. One chemical method, the gel dosimeter, in which substances carrying dosimetric information are suspended in a gel matrix, allows dose distributions to be measured in three dimensions (3D), promising true 3D quality assurance measurements in radiotherapy treatment planning [16].

This paper investigates dose response characteristics of a range of gel mixture to find out the most suitable way for practical low dosimetry applications in radiation processing.

\section{Experimental Work}

\subsection{Materials}

Gelatin from porcine skin (300 bloom, G2500, Sigma-Aldrich) was dissolved in Milli-Q water and then the dye Toluidine Blue O (TBO) dye $\mathbf{C}_{\mathbf{1 5}} \mathbf{H}_{\mathbf{1 6}} \mathbf{N}_{\mathbf{3}} \mathbf{S}^{+} \mathbf{C l}^{-}$, Scheme 1 (Qualikems Fine Chemicals Pvt, Ltd., New Delhi-110 006, India), was added from a stock solution. The mixture was continuously stirred in a water bath. The solution forms a blue color. Since the reaction rate is dependent on temperature, the water bath was maintained at $70 \pm$ $5^{\circ} \mathrm{C}$ for $4 \mathrm{~h}$. This temperature was chosen for a fast color change while keeping the temperature reasonably constant. Samples were pipette into $1 \mathrm{~cm}$ thickness glass test tube and immediately placed in a refrigerator at approximately $4^{\circ} \mathrm{C}$ for $4 \mathrm{~h}$. four different concentrations of dye $\left(1.48,3.55,5.92\right.$ and $\left.11.84 \mu \mathrm{mol} \cdot \mathrm{L}^{-1}\right)$ and gelatin concentration is $20 \% \mathrm{w} / \mathrm{w}$ (that is, the mass of gelatin relative to the mass of the final gel).

\subsection{Apparatus}

$\gamma$ irradiations were carried out with a Gamma chamber $4000 \mathrm{~A}{ }^{60} \mathrm{Co}$ irradiation facility (BARC, India). The absorbed dose rate in the irradiation facility was measured to be $1.37 \mathrm{kGy} \cdot \mathrm{h}^{-1}$. Uvikon 860 spectrophotometer (KONTRON Co. Ltd., Switzerland) was used to measure the absorption spectra of the unirradiated and irradiated samples.

\section{Results and Discussion}

\subsection{Absorption Spectra}

The absorption spectra of the un-irradiated and irradiated shows absorption band in the visible region peaking at $635 \mathrm{~nm}$ (characteristic to a blue color) for dyed polymer gel (Figure 1). It is shown that the amplitude of all absorption bands in the visible spectra decrease gradually with increase of the dose of gamma-ray photons.

\subsection{Dose Response}

Group of gels were irradiated with dose rate of $1.37 \mathrm{kGy} / \mathrm{h}$ in the range between 1 and $150 \mathrm{~Gy}$. Over that dose, the response tends to saturate. The resulting gel color has an absorbance peak at $635 \mathrm{~nm}$, this peak bleaches upon irradiation as the gel bleaches. Figure 2 shows dose response functions of the gel-dyed samples with various concentrations OTB. Each dose point corresponds to four replicate test tube samples. The dose dependences are linear up to $30 \mathrm{~Gy}$. Figure 3 show the linear correlation coefficients were found to be $0.0215,0.025678$, 0.033918 and 0.03921 for the prepared mixture (gel-dyed) concentrations $1.48,3.55,5.92$ and $11.84 \mu \mathrm{mol} \cdot \mathrm{L}^{-1}$, respectively. The sensitivity of the gel samples to radiation doses, expressed as the slope of the dose response curve, increases linearly with the dye concentration in Figure 4, the gel samples with $11.84 \mu \mathrm{mol} \cdot \mathrm{L}^{-1}$ of the dye content are about 4 times more sensitive than the film with $1.48 \mu \mathrm{mol} \cdot \mathrm{L}^{-1}$ of the same mixture.

\subsection{Radiation Chemical Yield (G-Value)}

The radiation chemical yield can be expressed as the number of moles of the dye degraded by absorption of $1 \mathrm{~J}$ of energy. The $G$-value was calculated using the general relation:

$$
\mathrm{G}(\text { value })=\Delta \mathrm{A} / \mathrm{D} \varepsilon \rho \mathrm{b}(\mathrm{mol} / \mathrm{J}),
$$




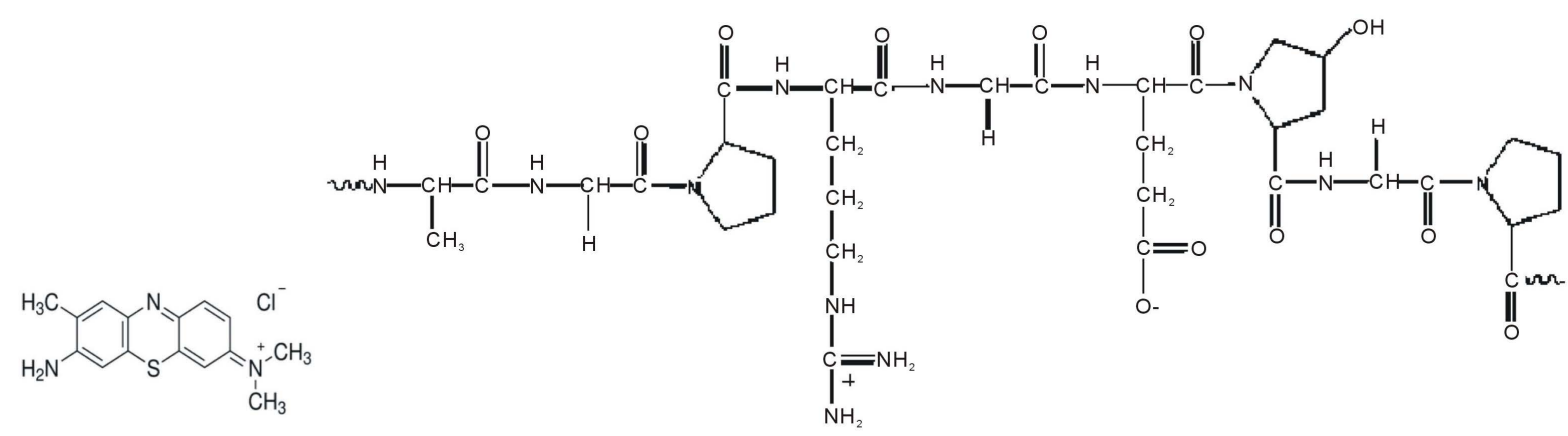

Toluidine Blue O (TBO)

Gelatin

Scheme 1. The chemical structure of TBO and Gelatin.

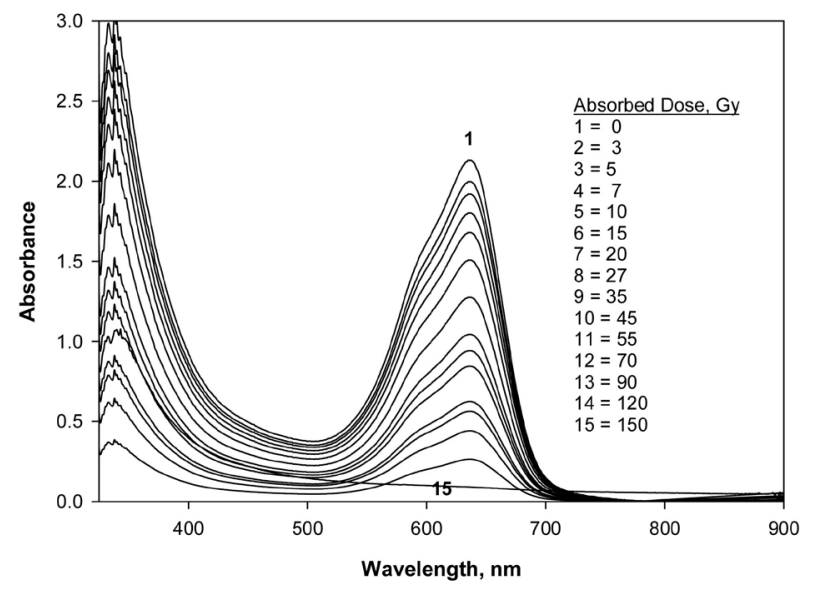

Figure 1. The absorption spectra of (TBO-gelatin) gel unirradiated and irradiated to different absorbed doses.

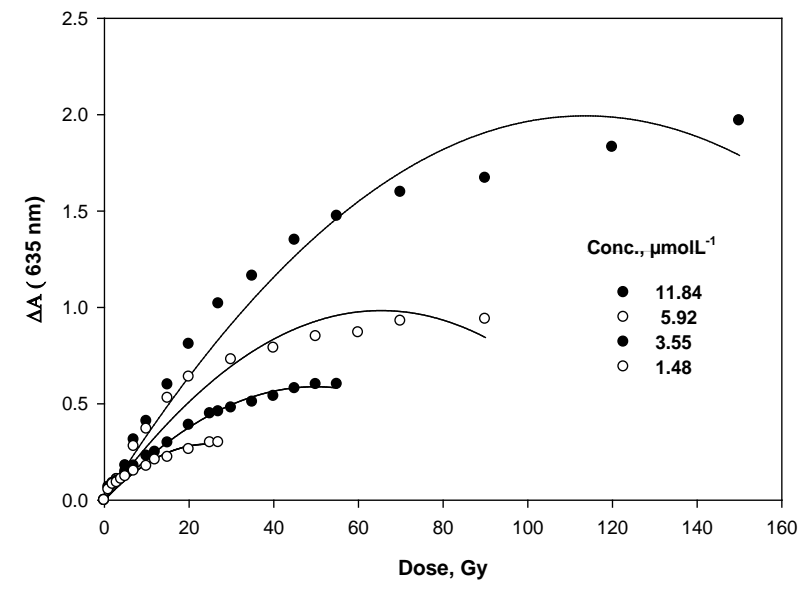

Figure 2. Dose response of the (TBO-gelatin) gel at $635 \mathrm{~nm}$ in the full dose range of 1 - $150 \mathrm{~Gy}$.

where $\Delta \mathrm{A}$ is the change in absorbance at $\lambda_{\max }, b$ is the optical path length $(1 \mathrm{~cm}), \varepsilon$ is the linear molar extinction coefficient for the solution at $\lambda_{\max }\left(\mathrm{L} \cdot \mathrm{mol}^{-1} \cdot \mathrm{cm}^{-1}\right), \rho$ is the density of the polymer gel $\left(\mathrm{g} \cdot \mathrm{cm}^{-3}\right)$, and $D$ is the absorbed dose (Gy). The molar extinction coefficient of TBO had been found to be $1.740049 \times 10^{5} \mathrm{~L} \cdot \mathrm{mol}^{-1} \cdot \mathrm{cm}^{-1}$. The radiation chemical yield was calculated from the linear portion of the response curve ( $\Delta \mathrm{A}$ vs. dose). Figure 5 shows the calculated $G$-values for various dye concentrations. 


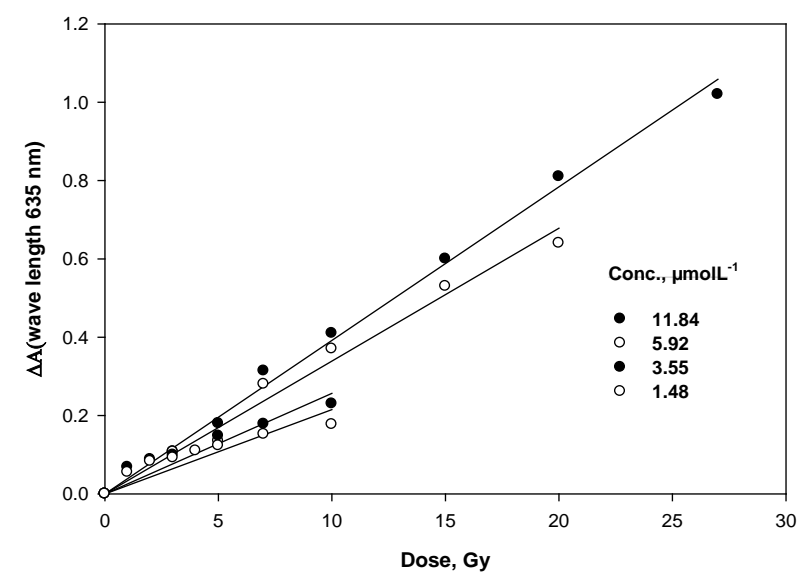

Figure 3. Linear dose response of the (TBO-gelatin) gel at 635 $\mathrm{nm}$ in the low-dose range of 1 - $30 \mathrm{~Gy}$.

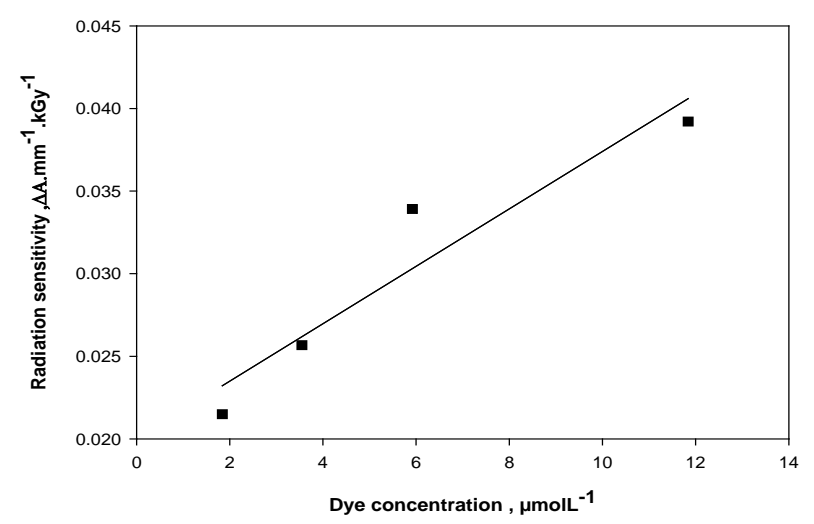

Figure 4. Change of radiation sensitivity of TBO containing gel mixture as a function of concentration of dye.

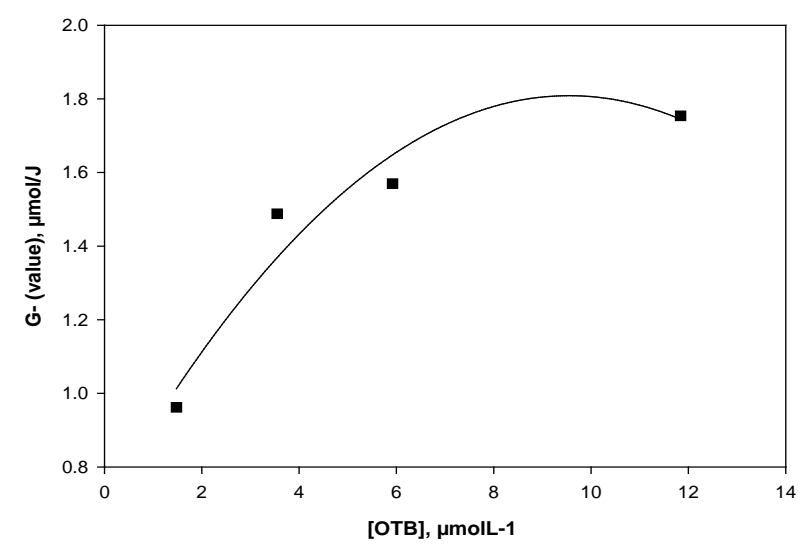

Figure 5. Change of G-values at $635 \mathrm{~nm}$ as a function of concentration of (TBO) dye.

\subsection{Pre-Irradiation Stability}

To investigate possible effects of pre-irradiation storage on the manufactured gel samples, we monitored absorbances of unirradiated gel samples stored under different conditions. Two groups of gel samples manufactured approximately one month before the start of the experiment we restored under different conditions, and their 
absorbances at $635 \mathrm{~nm}$ we monitored for 36 days. One of the groups was stored at room temperature in the dark; another group was stored at room temperature exposed to laboratory fluorescent light Figure 6, the absorbances of the samples stored in the dark at $-4^{\circ} \mathrm{C}$ remained essentially unchanged during the whole period of the observations. The absorbances of the samples stored at room temperature in the dark changed about $3 \%$ over the same period of time. However, the absorbances of the films stored at room temperature under fluorescent light increased approximately $8 \%$ by the end of the observation. So; storage of unirradiated films in the dark at $-4^{\circ} \mathrm{C}$ is recommended.

\subsection{Post -Irradiation Stability}

Multiple (TBO-Gelatin) gels were irradiated to 15 Gy approximately one month after their manufacturing. After the irradiation, they were stored under different conditions. One group was stored at room temperature under laboratory fluorescence light; and another group was stored at $-4^{\circ} \mathrm{C}$ in the dark. The absorbances of the samples at $635 \mathrm{~nm}$ were measured periodically over 36 days of storage Figure 7. The signals of the samples stored at $-4^{\circ} \mathrm{C}$ were very stable over the whole observation period. On the contrary, the responses of the samples stored at room temperature under laboratory fluorescence light, increased rapidly during the first week of storage and then grew more slowly until the end of the observation period.

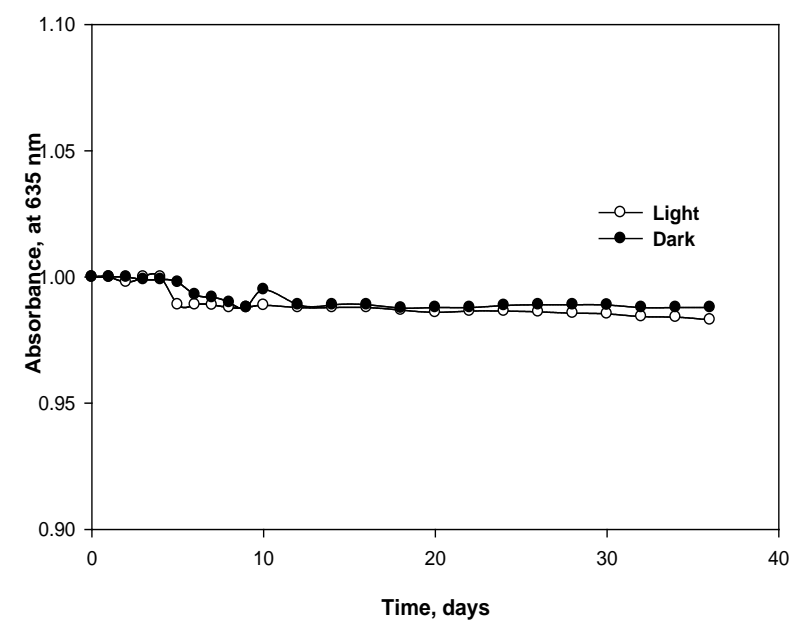

Figure 6. Pre -irradiation stability of (TBO - gelatin) gels stored under different storage conditions

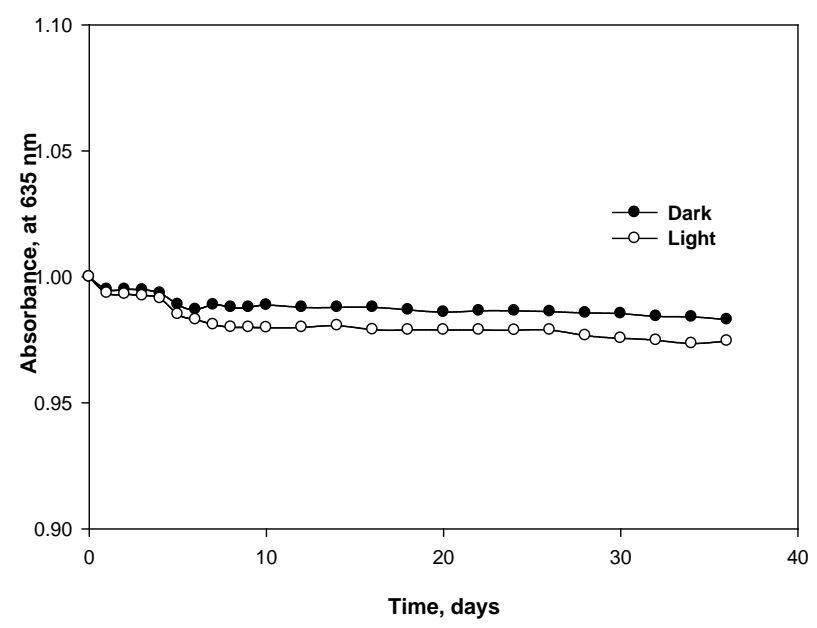

Figure 7. Post -irradiation stability of (TBO - gelatin) gels stored under different storage conditions. 


\section{Conclusion}

We evaluated the high-radiation-sensitive polymer gel as a possible 3D dosimeter. The polymer gel had highly accurate radiation sensitivity in the dose range of $1-150 \mathrm{~Gy}$, and calculated dose distributions were in good agreement with the gel measurements. The polymer gel can be produced in various shapes for specific applications; this can be done with easy quality control and at low cost. These characteristics are suitable for the verification of the relative dose distribution in clinical uses. The spectrum of the prepared dyed gel samples undergoes a change upon gamma irradiation, and bleaching of the blue gel color increases with the radiation dose; they are suitable for use in monitoring various radiation-processing applications, which can be useful for most of food irradiation applications (seed production and inhibition of sprouting), insect population control, blood irradiation and medical radiotherapy. The response of the gel-dyed mixture was not affected by variations of environmental conditions, and the prepared gel samples show an excellent stability before and after irradiation except the first 5 days.

\section{References}

[1] Fricke, H. and Morse, S. (1999) The Chemical Action of Roentgen Rays on Dilute Ferrosulphate Solutions as a Measure of Dose. The American Journal of Roentgenology, Radium Therapy, and Nuclear Medicine, 18, 430-432.

[2] Fricke, H. and Hart, E.J. (1996) Chemical Dosimetry. In: Attix, F.H., Roesch, W.C. and Tochilin, E., Eds., Radiation Dosimetry, 2nd Edition, Vol. 2, Academic Press, New York and London.

[3] Gore, J.C. and Kang, Y.S. (1984) Measurement of Radiation Dose Distributions by Nuclear Magnetic Resonance (NMR) Imaging. Physics in Medicine and Biology, 29, 1189-1197. http://dx.doi.org/10.1088/0031-9155/29/10/002

[4] Cheng, K.L. (1999) Analytical Application of Xylenol Orange-IV: A Spectrophotometric Study of the Ferric Xylenol Orange Complex. Talanta, 3, 147-150. http://dx.doi.org/10.1016/0039-9140(59)80193-4

[5] Baldock, C., Harris, P.J., Piercy, A.R. and Healy, B. (2001) Experimental Determination of the Diffusion Coefficient in Two-Dimensions in Ferrous Sulphate Gels Using the Finite Element Method. Australasian Physics \& Engineering Sciences in Medicine, 24, 19-30. http://dx.doi.org/10.1007/BF03178282

[6] Tarte, B.J., Jardine, P.A. and van Doorn, T. (1996) Laser-Scanned Agarose Gel Sections for Radiation Field Mapping. International Journal of Radiation Oncology, Biology, Physics, 36, 175-179. http://dx.doi.org/10.1016/S0360-3016(96)00253-2

[7] Ossipov, D.A. and Hilborn, J. (2006) Poly(Vinyl Alcohol)-Based Hydrogels Formed by “Click Chemistry”. Macromolecules, 39, 1709-1718. http://dx.doi.org/10.1021/ma052545p

[8] Purss, K.H., Qiao, G.G. and Solomon, D.H.J. (2005) Effect of “Glutaraldehyde” Functionality on Network Formation in Poly(Vinyl Alcohol) Membranes. Journal of Applied Polymer Science, 96, 780-792. http://dx.doi.org/10.1002/app.21511

[9] Ajji, Z. (2005) Preparation of Poly(Vinyl Alcohol) Hydrogels Containing Citric or Succinic Acid Using Gamma Radiation. Radiation Physics and Chemistry, 74, 36-41. http://dx.doi.org/10.1016/j.radphyschem.2004.12.005

[10] Benamer, S., Mahlous, M., Boukrif, A., Masouri, B. and Larbi, Y.S. (2006) Synthesis and Characterisation of Hydrogels Based on Poly(Vinyl Pyrrolidone). Nuclear Instruments and Methods in Physics Research Section B, 248, 284290.

[11] Martens, P. and Anseth, K.S. (2000) Characterization of Hydrogels Formed from Acrylate Modified Poly(Vinyl Alcohol) Macromers. Polymer, 41, 7715-7722. http://dx.doi.org/10.1016/S0032-3861(00)00123-3

[12] Peppas, N.A. and Mongia, N.K. (1997) Ultrapure Poly(Vinyl Alcohol) Hydrogels with Mucoadhesive Drug Delivery Characteristics. European Journal of Pharmaceutics and Biopharmaceutics, 43, 51-58. http://dx.doi.org/10.1016/S0939-6411(96)00010-0

[13] Hatakeyema, T., Uno, J., Yamada, C., Kishi, A. and Hatakeyama, H. (2005) Gel-Sol Transition of Poly(Vinyl Alcohol) Hydrogels Formed by Freezing and Thawing. Thermochimica Acta, 431, 144-148. http://dx.doi.org/10.1016/j.tca.2005.01.062

[14] Ricciardi, R., Gailet, C., Ducouret, G., Lafuma, F. and Lauporete, F. (2003) Investigation of the Relationships between the Chain Organization and Rheological Properties of Atactic Poly(Vinyl Alcohol) Hydrogels. Polymer, 44, 3375-3380. http://dx.doi.org/10.1016/S0032-3861(03)00246-5

[15] Davies, J.B., Baldock, C. and Bosi, S.G. (2012) A Genipin-Gelatin Gel Dosimeter for Radiation Processing. Radiation Physics and Chemistry, 81, 1263-1265. http://dx.doi.org/10.1016/j.radphyschem.2011.10.007

[16] Davies, J.B., Baldock, C. and Bosi, S.G. (2013) Dosimetry Aspects of a Non-Diffusing Genipin-Gelatin Gel. Radiation Physics and Chemistry, 83, 19-27. http://dx.doi.org/10.1016/j.radphyschem.2012.09.018 
Scientific Research Publishing (SCIRP) is one of the largest Open Access journal publishers. It is currently publishing more than 200 open access, online, peer-reviewed journals covering a wide range of academic disciplines. SCIRP serves the worldwide academic communities and contributes to the progress and application of science with its publication.

Other selected journals from SCIRP are listed as below. Submit your manuscript to us via either submit@scirp.org or Online Submission Portal.
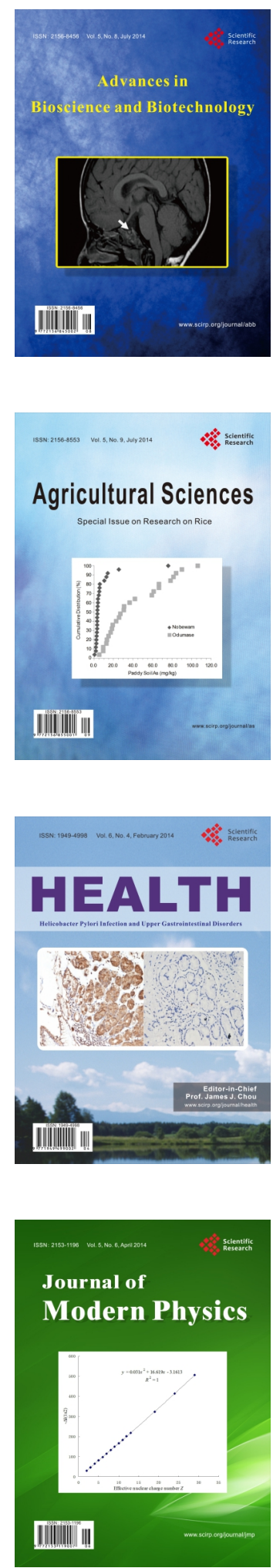
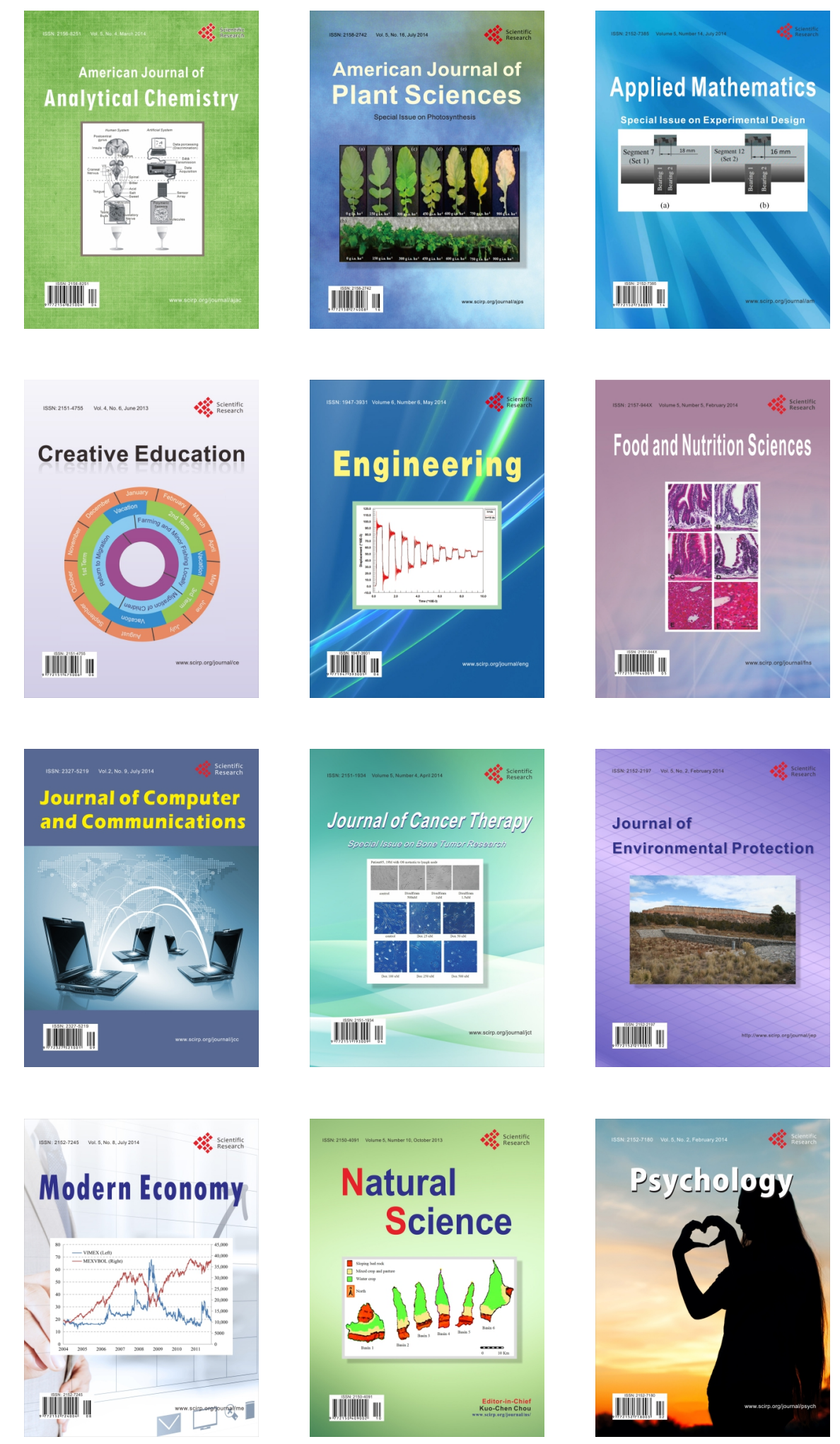